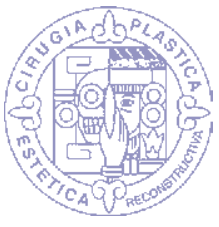

TrABAjO DE INVESTIGACIÓN

doi: $10.35366 / 91714$

https://dx.doi.org/10.35366/91714

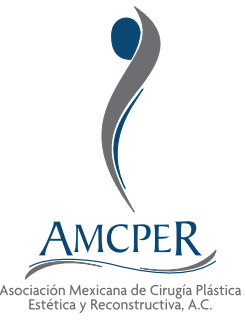

\title{
Tratamiento quirúrgico de la oreja constreñida
}

\author{
Surgical treatment for constricted ear
}

\author{
Dra. Cynthia Ivette Euán-Vázquez, ${ }^{*}$ Dr. Rodrigo Dávila-Díaz, \\ Dr. Raúl Alfonso Vallarta-Rodríguez,* Dr. Cuauhtémoc Márquez-Espriella, $\$$ \\ Dr. Arnoldo Otoniel Topete-González," Dra. Priscila Rojas-García"
}

Palabras clave: Microtia congénita, oreja constreñida, oído externo, pabellón auricular, cartílago, anormalidades congénitas.

Keywords: Congenital microtia constricted ear, external ear, auricular pavilion, cartilage, congenital abnormalities.

\footnotetext{
* Cirujano plástico y
} reconstructivo. Hospital Médica Sur.

‡ Cirujano plástico y reconstructivo.

Servicio de Cirugía Reconstructiva. Hospital Central Sur de Alta Especialidad PEMEX. $\S$ Cirujano plástico y reconstructivo. Jefe de Servicio de Cirugía Reconstructiva. Hospital Central Sur de Alta Especialidad PEMEX. ${ }^{\|}$Residente del $4 .^{\circ}$ año de Cirugía Plástica y Reconstructiva. Hospital Central Sur de Alta Especialidad PEMEX.

^ Cirujana plástica y reconstructiva. Centro Médico ABC Campus Santa Fe.

Los autores de este artículo no tienen conflicto de intereses qué declarar.

\section{RESUMEN}

Presentamos los resultados postoperatorios de pacientes con diagnóstico de oreja constreñida, sometidos a reconstrucción auricular, de acuerdo con la clasificación de Tanzer para oreja constreñida, en el Hospital Central Sur de Alta Especialidad de PEMEX, de marzo de 2016 a marzo de 2017. Incluye pacientes a los que se realizó reconstrucción auricular por diagnóstico de oreja constreñida, efectuada por el mismo cirujano en un solo tiempo quirúrgico, utilizando o no cartílago costal. Evaluamos los resultados mediante análisis subjetivos de la anatomía del tercio superior por medio de la escala de Likert a la semana, al mes, tres, seis y 12 meses. En un año se intervino un total de seis orejas en cinco pacientes, con promedio de edad de 12.8 años: tres hombres y dos mujeres; $50 \%$ con oreja constreñida Tanzer II-B. En $80 \%$ utilizamos cartílago costal sin complicaciones. Dos pacientes requirieron una segunda intervención para realizar otoplastia y simetrizar la oreja contralateral. Todos tuvieron un seguimiento de 12 meses, se observó un excelente resultado en tres pacientes y bueno en dos. Concluimos que la oreja constreñida es una anormalidad congénita subdiagnosticada en nuestro país, la cual amerita una evaluación individualizada para determinar el tratamiento reconstructivo que dé resultados satisfactorios a largo plazo, sin que se requiera un segundo tiempo quirúrgico.

\section{ABSTRACT}

We present the postoperative results of patients with a diagnosis of constricted ear undergoing atrial reconstruction, according to the Tanzer classification for constricted ear, at the PEMEX Central South High Specialty Hospital, from March 2016 to March 2017. It includes patients who had ear reconstruction by diagnosis of a constricted ear, performed by the same surgeon in a single surgical time, using or not using costal cartilage. We evaluated the results by subjective analysis of the anatomy of the upper third by means of the Likert scale at one week, one month, three, six and 12 months. In a year, a total of six ears were operated on five patients, with an average age of 12.8 years: three men and two women; 50\% with constricted ear Tanzer IIB. In $80 \%$ of those cases, we used costal cartilage without complications. Two patients required a second intervention to perform otoplasty and symmetrize the contralateral ear. All were followed up for 12 months, observing an excellent result in three patients and good in two. We conclude that the constricted ear is an underdiagnosed congenital abnormality in our country that merits an individualized evaluation to determine the reconstructive treatment that provides satisfactory longterm results, without requiring a second surgery.

\section{INTRODUCCIÓN}

$\mathrm{E}_{y}^{\prime}$ pabellón auricular se forma entre la quinta y novena semanas a partir de seis proliferaciones mesenquimatosas, situadas en los extremos dorsales del primero y del segundo arco faríngeo que rodean la primera hendidura faríngea. ${ }^{1}$ La fusión de estas prominencias auriculares es complicada, por lo que no es raro que se produzcan malformaciones del desarrollo. ${ }^{1,2}$
El oído y el conducto auditivo externos son muy sensibles a la exposición durante el primer trimestre a fármacos como estreptomicina, talidomida y salicilatos que causan agenesia o atresia en estas dos estructuras. ${ }^{3}$ McKenzie y colaboradores reportan que la causa de las anormalidades del desarrollo auricular es la isquemia del tejido uterino, resultado de una obliteración de la arteria estapedia o de hemorragia de los tejidos locales. ${ }^{4}$ 
Recibido:

05 febrero 2019

Aceptado para publicar: 26 junio 2019
En México, el registro y vigilancia epidemiológica de malformaciones congénitas externas reveló una prevalencia de microtia de $7.37 / 10,000$ nacidos vivos y muertos durante el año 2010. ${ }^{5}$

La microtia se clasifica según el área anatómica afectada y una de ellas es la oreja constreñida, que afecta el tercio superior y corresponde a la clasificación IV-A de Tanzer. 6,7

En 1975, Tanzer $^{8}$ clasificó las orejas constreñidas en:

I. Colapso del hélix únicamente.

II-A. Deficiencia de la escafa, crural superior y fosa triangular con colapso del hélix superior, dando como resultado pérdida de la altura vertical, protrusión y doblez (forma de tapa). El tratamiento quirúrgico no requiere injerto de piel para expandir el margen auricular.

II-B. Mismas características clínicas que el II-A sin antihélix ni crural, con reducción considerable de la altura. El tratamiento quirúrgico requiere injerto de piel para expandir el margen auricular.

III. Fijación anterior del hélix cerca del lóbulo, la aurícula está en forma de bolsa y la oreja por lo regular tiene una implantación baja.

La oreja constreñida es un grupo de anomalías auriculares del tercio superior de la oreja, en donde parece que su borde ha sido apretado como un cordón. ${ }^{7,8}$ Posee cuatro características: forma de tapa, disminución de la altura vertical, protrusión y posición baja de la oreja. La reconstrucción auricular depende del grado de severidad de la hipoplasia. ${ }^{8,9}$

Para efectos quirúrgicos, la única diferencia entre la oreja del recién nacido y del adulto radica en que el cartílago del neonato es más maleable y suave. ${ }^{10,11}$ La anatomía es la misma; tiene $85 \%$ del desarrollo de la oreja del adulto a la edad de tres años. ${ }^{11,12} \mathrm{El}$ ancho de la oreja es aproximadamente de 55 a $60 \%$ de alto. ${ }^{13}$

En la reconstrucción auricular, el uso experimental y clínico del cartílago costal autólogo que reportó Pierce, a principios de 1930, aportó una nueva técnica. A pesar de que muchos materiales se han utilizado para la reconstrucción del marco auricular, el car- tílago autólogo se considera como el material estándar. ${ }^{14}$

El objetivo de este estudio es mostrar los resultados postoperatorios de la reconstrucción auricular de pacientes que fueron diagnosticados con oreja constreñida, en el Hospital Central Sur de Alta Especialidad de PEMEX, y a quienes se les realizó la reconstrucción quirúrgica con el uso o no de cartílago costal autólogo, de acuerdo a la evaluación y clasificación de Tanzer para oreja constreñida. ${ }^{8}$

\section{MATERIAL Y MÉTODO}

Para este trabajo se contó con la aprobación del Comité de Ética e Investigación del Hospital Central Sur de Alta Especialidad de PEMEX, con los datos personales de los pacientes de manera confidencial y con la autorización quirúrgica mediante consentimiento informado.

Se incluyó a todos con malformación auricular congénita de oreja constreñida, ${ }^{8}$ de cualquier género y grupo de edad que aceptaron mediante consentimiento informado recibir tratamiento quirúrgico reconstructivo. El procedimiento fue realizado por el mismo cirujano y ayudante en el periodo del 01 de marzo de 2016 al 01 de marzo de 2017. Los pacientes diagnosticados con este tipo de microtia se subdividieron en grupos de acuerdo a la clasificación de Tanzer de oreja constreñida, ${ }^{8}$ en I, II-A, II-B, o III y, conforme a ésta, se decidió

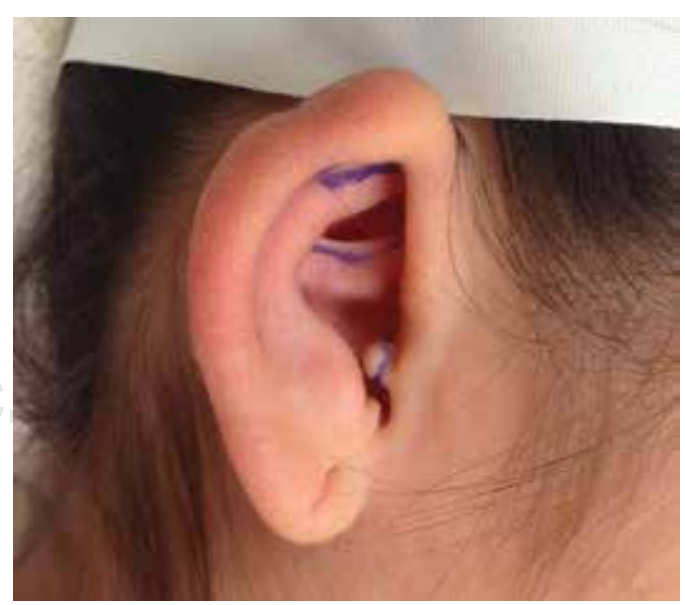

Figura 1: Paciente de 11 años con oreja constreñida derecha Tanzer IIB. 


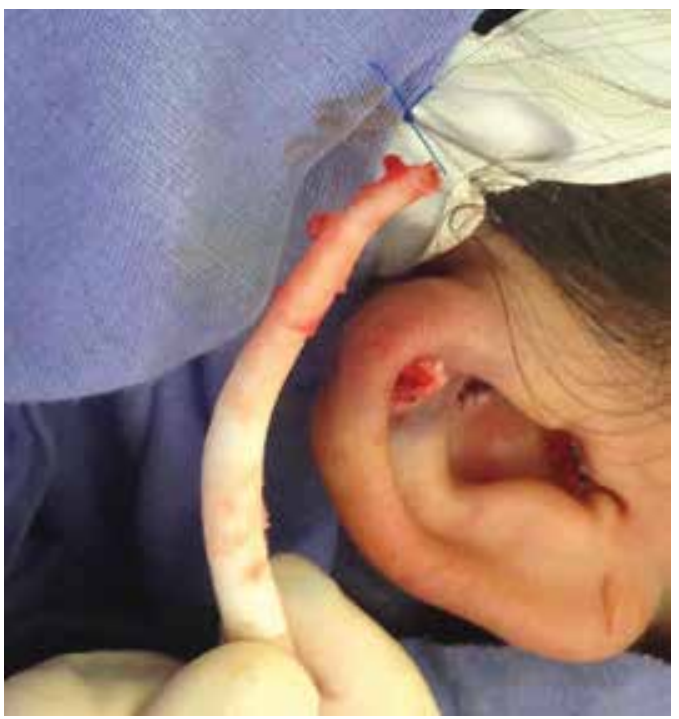

Figura 2: Transoperatorio de la reconstrucción auricular con cartílago costal autólogo mediante acceso anterior.

utilizar o no cartílago costal autólogo durante la reconstrucción. Además, se registraron variables independientes como edad, género, lado afectado y tipo de presentación, si era aislada o sindromática; también variables dependientes como la existencia de intervención quirúrgica secundaria, el seguimiento en meses, complicaciones relacionadas con el procedimiento quirúrgico y si se utilizó o no cartílago costal para la reconstrucción.

Técnica quirúrgica. Reconstrucción en un solo tiempo (descrita para el grupo II de Tanzer $^{8}$ ). Cuando se detecta el doblez del hélix, se debe diferenciar entre los casos en los que el borde del hélix puede forzarse manualmente a volver a su forma normal, y los casos en los que el colapso y la adherencia del cartílago impiden regresar a la apariencia normal. De hecho, esto se puede deber a la carencia de la crural superior del antihélix, para dar soporte al borde del hélix. El acceso es a través de una incisión anterior en la piel dentro del borde del hélix (Figura 1), o en la parte posterior del pabellón auricular para movilizar la piel en la región de la escafa, hasta que la parte superior del antihélix esté completamente expuesta.

El acceso anterior es conveniente, porque la cicatriz resultante se oculta bien dentro del borde del hélix. La altura del injerto costal se elige donde el borde del hélix tenga relieve y la altura de la oreja se iguale al lado no deforme (Figura 2). El cartílago costal da soporte al borde del hélix y previene el hundimiento; se cosecha de la quinta costilla a través de una incisión inframamaria de 2 a $3 \mathrm{~cm}$ y se debe adelgazar a $1.5 \mathrm{~mm}$.

El cartílago costal ya moldeado se fija al borde del hélix con dos suturas de nylon 6-0 y con el segundo par se sutura a la transición del antihélix y la crural inferior. La piel se cierra con nylon 5-0 y se cubre con gasas impregnadas con petrolato y vendaje no opresivo por 24 horas. No se dejan drenajes (Figura 3).

En los casos en los que el doblez se pueda corregir manualmente (Tanzer IIA), no se requiere escisión del cartílago costal (Figura 4). De acuerdo con el caso de adherencia, el acceso es anterior o posterior, y la escisión puede ser en forma de media luna. Si presenta una escafa deforme, es necesario movilizar el borde del hélix hacia una posición más cefálica, se moldea el antihélix y/o escafa fijándolo con puntos de nylon 6-0 para después afrontar la piel con nylon 5-0 (Figura 5).

El seguimiento postoperatorio se dio a la semana para retirar los puntos y se evaluó la herida quirúrgica al mes, tres, seis y 12 meses para control fotográfico. Se evaluaron los resultados mediante análisis subjetivo de la anatomía del tercio superior por subunidades estéticas de dicha región, de acuerdo a las siguientes variables:

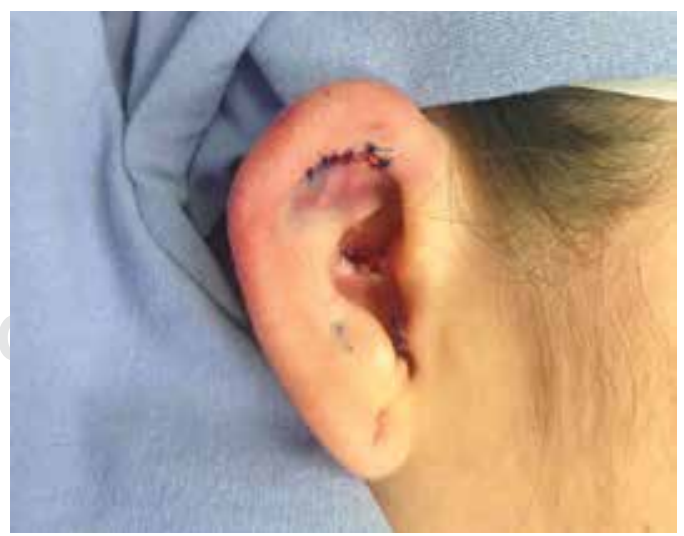

Figura 3: Postoperatorio inmediato de la reconstrucción con cartílago costal en oreja constreñida Tanzer IIB. 


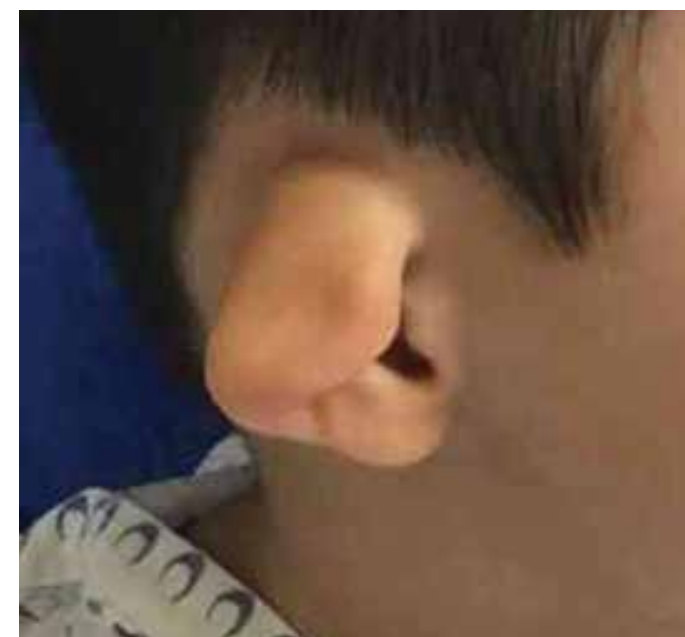

Figura 4: Preoperatorio paciente Tanzer IIA.

- Sitio donador

- Definición del hélix

- Definición del antihélix

- Escafa

- Fosa triangular

- Posición de la oreja

Esta evaluación subjetiva se realizó en el periodo comentado previamente por tres cirujanos plásticos, adscritos al Servicio de Cirugía Plástica y Reconstructiva y ajenos al procedimiento quirúrgico, mediante cuestionarios con escala de Likert: 1 (mal resultado), 2 (regular resultado), 3 (buen resultado) y 4 (excelente resultado).

\section{RESULTADOS}

Durante un año se intervinieron cinco pacientes (tres hombres y dos mujeres), con edad de tres a 25 años (media 12.8 años). El lado afectado fue el derecho en cuatro pacientes $(80 \%)$ y bilateral en un paciente (20\%). Seis orejas intervenidas en total, con presentación aislada en cuatro (80\%) y sindromática en uno (20\%) (síndrome de Kabuki); cirugías secundarias en dos pacientes (40\%) después de los 12 meses para simetría contralateral (otoplastia). En cuatro pacientes $(80 \%)$ se utilizó injerto costal para la reconstrucción (Figura 2). Se llevó a cabo el seguimiento a 12 meses en $100 \%$, ninguno tuvo complicaciones (Tabla 1).
Los resultados de la evaluación subjetiva de los controles fotográficos mediante la escala de Likert (Tabla 2), de acuerdo al seguimiento a los 12 meses, se calificaron como excelente en tres pacientes (60\%) (Figura 6) y bueno en $\operatorname{dos}(40 \%)$.

De las seis orejas intervenidas, tres (50\%) se clasificaron como Tanzer IIB (Figura 1), dos (33\%) como Tanzer IIA (Figura 4) y una (17\%) como Tanzer III (Figura 7).

\section{DISCUSIÓN}

La oreja constreñida (microtia tipo IV) es una anomalía que afecta al tercio superior del pabellón auricular; las variantes anatómicas son complejas en la mayoría de los casos, ${ }^{8}$ por lo que los resultados reconstructivos dependen de la valoración inicial para determinar el tipo de tratamiento quirúrgico que amerita. $^{4}$

En nuestra entidad, este tipo de malformación auricular no se trata en la mayoría de los casos, porque no tiene una presentación clínica severa, ya que no estamos diagnosticando una anotia (microtia Tanzer I) o una microtia Tanzer IIA, que son clínicamente más severas.

Algunos de los defectos del tercio superior pueden cerrarse de manera primaria sólo con tejidos blandos, pero el uso de cartílago se necesita con frecuencia para dar soporte. Para

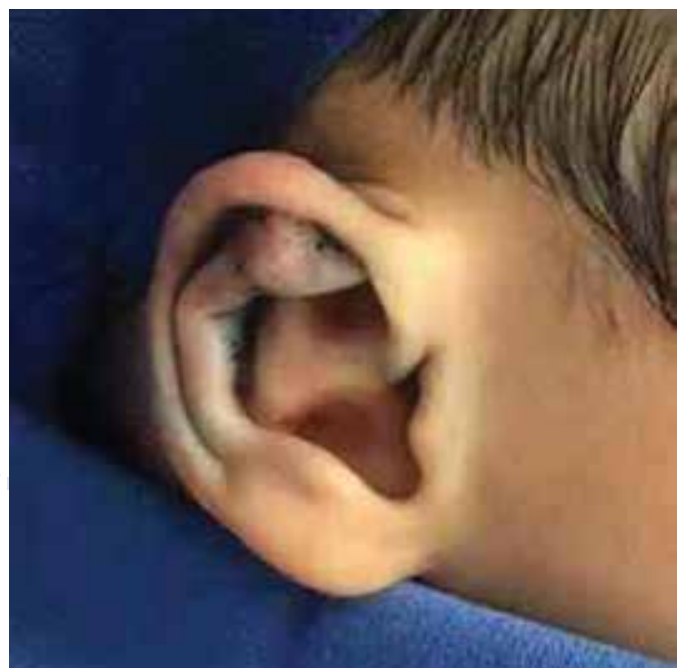

Figura 5: Postoperatorio inmediato de reconstrucción sin cartílago costal con acceso posterior. 


\begin{tabular}{lccccccc}
\multicolumn{7}{c}{ Tabla 1: Resultados de variables dependientes e independientes. } \\
Sexo & $\begin{array}{c}\text { Edad } \\
\text { (años) }\end{array}$ & $\begin{array}{c}\text { Lado } \\
\text { afectado }\end{array}$ & $\begin{array}{c}\text { Presentación } \\
\text { clínica }\end{array}$ & $\begin{array}{c}\text { Complica- } \\
\text { ciones }\end{array}$ & $\begin{array}{c}\text { Corrección } \\
\text { secundaria }\end{array}$ & $\begin{array}{c}\text { Uso de } \\
\text { cartílago costal }\end{array}$ & $\begin{array}{c}\text { Seguimiento } \\
\text { (meses) }\end{array}$ \\
\hline Femenino & 12 & Derecho & Aislada & No & No & Sí & 12 \\
Masculino & 25 & Derecho & Aislada & No & Sí & Sí & 12 \\
Femenino & 11 & Derecho & Aislada & No & No & Sí & 12 \\
Masculino & 13 & Derecho & Sindromática & No & Sí & Sí & 12 \\
Masculino & 3 & Bilateral & Aislada & No & No & No & 12 \\
\hline
\end{tabular}

defectos pequeños, el injerto de cartílago de concha puede ser suficiente; sin embargo, para grandes defectos, las reglas de Firmin son extremadamente útiles: defectos de $25 \%$ o más del borde del hélix o si involucra más de dos planos (si involucra antihélix más hélix o escafa) requieren soporte con cartílago costal, ya que el cartílago de la concha no provee suficiente soporte en estos casos. ${ }^{15}$

En la oreja constreñida, la principal anormalidad es la deficiencia del borde del hélix en la circunferencia de la escafa, misma que se encuentra impactada. ${ }^{16}$ La inadecuada altura del hélix constreñido provoca que las fuerzas hagan que la oreja tome una forma de taza que sobresale de la cabeza, lo que resulta en una oreja ligeramente pequeña, por lo que cualquier intento de otoplastia se debe acompañar de un aumento de la altura del hélix. ${ }^{17}$

La reconstrucción auricular moderna se atribuye a Tanzer, por los principios detallados, la técnica y la evaluación crucial de la reconstrucción auricular total usó cartílago costal autólogo. Tanzer fue el parteaguas de la reconstrucción auricular en 1959, al realizar un marco auricular con un bloque de cartílago costal autólogo, sus excelentes resultados perduran hasta nuestros días. ${ }^{14} \mathrm{Es}$ por ello que decidimos tratar la oreja constreñida mediante el uso de injerto costal, si el caso lo ameritaba, de acuerdo a su clasificación, ya que en las referencias revisadas de oreja constreñida no se cuenta con reportes postoperatorios a largo plazo y la mayoría de las publicaciones describen tratamientos no quirúrgicos.

El tiempo y la selección del procedimiento quirúrgico se deben valorar cuando el paciente haya madurado, con el desarrollo apropiado de la oreja, tomando también en cuenta la carga del estrés psicosocial causado por la deformidad y la flexibilidad del cartílago auricular. Para minimizar en lo posible el estrés psicosocial, muchos cirujanos recomiendan intervenir antes de que el niño comience a socializar; sin embargo, en muchos casos se

\section{Tabla 2: Resultados de la evaluación subjetiva mediante escala de Likert.}

\begin{tabular}{cccccc} 
Paciente & 1 semana & 1 mes & 3 meses & 6 meses & 4 \\
1 & 3 & 3 & 4 & 4 & 4 \\
2 & 3 & 4 & 4 & 4 & 3 \\
3 & 3 & 4 & 3 & 4 & 3 \\
4 & 3 & 3 & 4 & 4 & 4 \\
\hline
\end{tabular}

(1) mal resultado, (2) regular resultado, (3) buen resultado, (4) excelente resultado. 


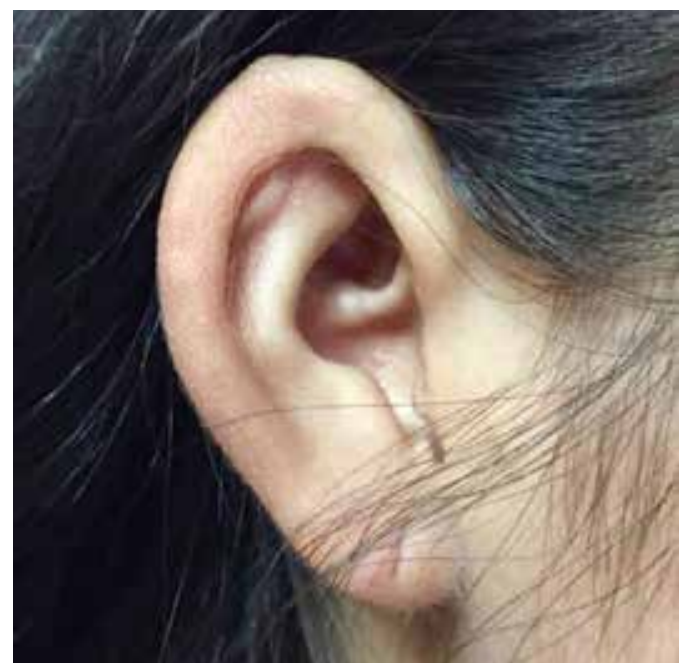

Figura 6: Control a los 12 meses de paciente con Tanzer IIB con resultado evaluado como excelente.

recomienda esperar a que el paciente sea lo suficientemente maduro para que participe de manera activa en su recuperación y cuidados postoperatorios. El desarrollo de la oreja llega a $85-90 \%$ a los tres años de edad, por lo cual decidimos incluir todas las edades de presentación en nuestro trabajo; no obstante, a los seis años el cartílago auricular comienza a estar más rígido, ${ }^{13}$ lo que hace más difícil moldear el cartílago en los adultos.

En un año, intervenimos a cinco pacientes con este diagnóstico, por lo que consideramos que es poco conocido y subdiagnosticado por los médicos de primer contacto, al desconocer que se trata de un subtipo de microtia que también se puede corregir quirúrgicamente o con conformadores, según sea la edad y la presentación clínica.

En nuestro país no se había descrito el tratamiento quirúrgico reconstructivo para esta anomalía congénita, ni la presentación clínica más frecuente, que en este caso resultó ser Tanzer IIB ${ }^{8}$ siendo el lado derecho el más afectado. Esto coincide con los reportes de publicaciones previas, donde se hace referencia que en la microtia el lado derecho es el más perjudicado, lo cual coincide con nuestro estudio.

Por otra parte, si seguimos el protocolo reconstructivo para oreja constreñida propuesto por Tanzer, ${ }^{8}$ se deberá usar injerto de piel en los tipos II-B o III; sin embargo, no fue necesario en ninguno de nuestros pacientes, pero sí lo fue recuperar la altura y la definición del antihélix con injerto costal autólogo en algunos de ellos.

En los casos descritos, los resultados en un año son de buenos a excelentes, colocando o no cartílago costal, lo que demuestra que la valoración inicial es determinante para la planeación quirúrgica, ya que según los hallazgos clínicos y transoperatorios que encontremos, decidiremos el uso o no de injerto costal.

Hacemos mención de cinco pacientes intervenidos en un año con el diagnóstico de oreja constreñida; sin embargo, es posible llegar a reportar mayor incidencia si se busca de manera intencionada, ya que por no presentar una malformación completa pasa desapercibida. El tratamiento reconstructivo se puede realizar con resultados satisfactorios en un solo tiempo quirúrgico.

\section{CONCLUSIONES}

La oreja humana es indispensable para la definición de la cara y es clave trascendental para su apariencia natural, armoniosa y estética. La reconstrucción del borde del hélix para dar una apariencia natural es difícil; los defectos del tercio superior se tratan quirúrgicamente, de acuerdo al tamaño y complejidad del mismo. Si se requiere aumentar la altura del hélix, se prefiere el uso de cartílago cos-

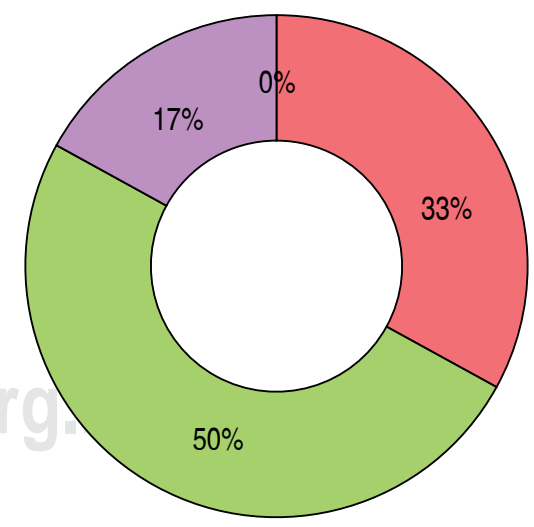

Tanzer IIA $\square$ Tanzer IIB $\square$ Tanzer III

Figura 7: Resultados de acuerdo a la clasificación de Tanzer para la oreja constreñida. 
tal, ya que ha dado resultados estéticos más satisfactorios en un solo tiempo quirúrgico, en comparación con el avance o rotación de colgajos únicamente.

La oreja constreñida es un tipo de microtia (microtia tipo IV) que afecta el tercio superior del pabellón auricular que, al no ser tan compleja como una anotia, en ocasiones pasa desapercibida, por lo que no puede ofrecerse un tratamiento quirúrgico reconstructivo.

En este estudio reportamos que el subtipo de oreja constreñida más común es Tanzer IIB. El lado derecho es el más afectado. Se presenta con más frecuencia en el sexo masculino y en forma clínica aislada. Cada presentación clínica amerita una evaluación individualizada para definir el tratamiento reconstructivo, con o sin el uso de injerto costal y así obtener resultados satisfactorios a largo plazo, sin que se requiera un segundo tiempo quirúrgico.

\section{REFERENCIAS}

1. Sadler TW. Oído. En: Sadler TW. Langman embriología médica con orientación clínica. $12^{a}$ ed. Philadelphia EUA: Lippincott Williams \& Wilkins 2016, pp. 321328.

2. Moore Keith L, Persaud TVN. Oído. En: Moore KL. Embriología clínica. 8ª ed. Madrid España: Elsevier Sauders 2013, pp. 430-433.

3. Carlson BM. Órganos de los sentidos: Oído. En: Carlson BM. Embriología humana y biología del desarrollo. 5a ed. Madrid, España: Elsevier Sauders 2014, pp. 286-293.

4. Neligan P. Reconstruction of the ear. In: Warren R, Neligan P. Plastic surgery. $3^{\text {rd }}$ ed. London: ElsevierSaunders 2013, pp. 245-311.

5. Aguinaga-Ríos M, Frías S, Arenas-Aranda DJ, MoránBarroso F. Microtia-atresia: aspectos clínicos, genéticos y genómicos. Bol Med Hosp Infant Mex 2014; 71 (6): 387-395.

6. Schonauer F, La Rusca I, Molea G. Non-surgical correction of deformational auricular anomalies. J Plast Reconstr Aesthet Surg 2009; 62 (7): 876-883. doi: 10.1016/j.bjps.2007.11.072.
7. Bi Y, Lin L, Yang Q, Surgical correction of constricted ear combined with Stahl's ear. J Plast Reconstr Aesthet Surg 2015; 68 (7): 895-901. doi: 10.1016/j. bjps.2015.03.026.

8. Kon M, van Wijk MP. T-bar reconstruction of constricted ears and a new classification. J Plast Reconstr Aesthet Surg 2014; 67 (3): 358-361. doi: 10.1016/j.bjps.2013.12.020.

9. Guyuron B, Eriksson E, Persing JA et al. Otoplasty. In: Guyuron B, Achauer B, Eriksson E, Russell R, Coleman J, VanderKolk C (eds). Plastic surgery: indications, operations, outcomes. St. Louis: Mosby, 2000.

10. Janis JE, Rohrich RJ, Gutowski KA. Otoplasty. Plast Reconstr Surg 2005; 115 (4): 60e-72e. doi: 10.1097/01.PRS.0000156218.93855.C9.

11. Schonauer F, Vuppalapati G, Marlino S, Santorelli A, Canta L, Molea G. Versatility of the posterior auricular flap in partial ear reconstruction. Plast Reconstr Surg 2010; 126 (4): 1213-1221. doi: 10.1097/ PRS.0b013e3181ec1f03.

12. Janz BA, Cole P, Hollier LH, Stal S. Treatment of prominent and constricted ear anomalies. Plast Reconstr Surg 2009; 124 (Suppl. 1): 27e-37e. doi: 10.1097/PRS.0b013e3181aa0e9d.

13. Bruker MJ, Patel J, Sullivan PK. A morphometric study of the external ear: age and sex-related differences. Plast Reconstr Surg 2003; 112 (2): 647-652.

14. Beahm EK, Walton RL. Auricular reconstruction for microtia: part I. Anatomy, embryology, and clinical evaluation. Plast Reconstr Surg 2002; 109 (7): 24732482; quiz following 2482. doi: 10.1097/00006534200206000-00100.

15. Thorne CH. CKC. Grabb and Smith's plastic surgery. 7th ed. Philadelphia, USA: Lippincott Williams \& Wilkins, 2014.

16. Bi Y, Lin L, Yang Q, Pan B, Zhao Y, He L et al. Surgical correction of constricted ear combined with Stahl's ear. J Plast Reconstr Aesthet Surg 2015; 68 (7): 895-901. doi: 10.1016/j.bjps.2015.03.026.

17. Thorne $\mathrm{CH}$, Wilkes G. Ear deformities, otoplasty and ear reconstruction. Plast Reconstr Surg 2012; 129 (4): 701e-716e. doi: 10.1097/PRS.0b013e3182450d9f.

Correspondencia:

Dra. Cynthia Ivette Euán Vázquez

Puente de Piedra Núm. 150, Consultorio 419-T2, Col. Toriello Guerra, 14050, Alcaldía Tlalpan, Ciudad de México, México.

E-mail: cynthiaeuan16@gmail.com 